incidence of infertile marriage which he said had been compiled by me. If Dr. Brown is correctly reported then his statement, with the implication that it indicates the present state of affairs, is misleading.

The statistics he quoted (incompletely and inaccurately in one respect) were apparently taken from my book. ${ }^{1}$ They are to be found in a paragraph which makes it clear that they apply not to the present or the immediate past but to the decade 19001909. This is before my time.-I am, etc.,

NORMAN JEFFCOATE

Department of Obstetrics and Gynaeco'ogy, The Un versity,

Liverpool

1 Jeffcoate, 'T. N. A., Principles of Gynaecology, 3rd edn., Butterworth, London, 1967.

\section{Mechanism of Action of Amantadine}

SIR,-It has been well established that amantadine is a moderately effective drug in patients with Parkinsonism. ${ }^{12}$ Little is known of its mode of action, and reports of side effects of hallucinosis, confusion, insomnia, and dizziness are so non-specific as to shed little extra light on the level or site of central action.

A 68-year-old lady with severe rigid and akinetic Parkinsonism, previously untreated, was given amantadine $100 \mathrm{mg}$ b.d. Her Webster score improved from $18 / 30$ to $14 / 30$ in one week, and six weeks later her score was $10 / 30$ and she was mobile and much less disabled. She complained, however, that "I can't keep my tongue still in my mouth," and examination confirmed a gross lingual-facial dyskinesia, precisely mimicking that seen with levodopa.

Amantadine has been shown in animal studies to potentiate catecholaminergic (including dopaminergic) transmission; this may be due to inhibition of neuronal uptake across the synaptic cleft, or it may release catecholamines from the small extragranular depots. ${ }^{3}$ Locomotor behaviour is increased to a much lesser extent than with amphetamines in animals, but of interest is the report $^{4}$ that it potentiates stereotyped behaviour (licking and gnawing) in rats and mice. This pattern of stereotyped motor behaviour has been related to dopamine activity, ${ }^{5}$ and the case summarized here may represent the first parallel to this observation in the human subject. The evidence suggests that the augmentation of dopaminergic transmission is more likely to be due to release from extragranular stores than from the larger granular stores, since amantadine has no effect on raising cerebrospinal fluid levels of homovanillic acid derived from dopamine breakdown.-I am, etc.,

JoHn Pearce

Combined Neurological Service, Hull Royal Infirmary,

Hull, Yorks

1 Parkes, J. D., et a!. Lancet, 1971, 1. 1083 Rao, N. S., and Pearce, J., Practitioner, 1971 3 Offermeier, I., Parkinson's Disease, Ed. G. Birdwood, S. Gilder, and C. A. S. Wink, London. Academic Press, 1971 , p. 85

Simon, P., Malatray, J., and Borssier, J. R. Fournal. 546 . 5 Ernst, A. M., and Smelik, P. G., Experientia
Back to Vesalius, 1970

SIR,-A great deal has been written in recent years about the importance of impact, relevance, and the integrative approach in medical education especially in that Cinderella of the basic sciences, gross anatomy. It is often argued that familiarity with the appearance of the parts of the body in the dissecting room is of little help in visualizing these structures in the living or for their identification at operation.

The interest and enthusiasm shown by the University of Iowa freshmen medical students at the routine demonstrations of fresh human placentae as part of their integrated course in gross anatomy and human embryology caused some surprise, since the interest seemed out of proportion to the importance of the gross structure of the organ in question. It was therefore decided, in the interest of the fresh approach as well as in the ever continuing quest for the new and the unusual, to go back to Vesalius.

The subject was a 65 -year-old male, whose previously deeded body became available when he died instantaneously after impact with a telegraph pole. He was received, unembalmed, very shortly after death, without a previous postmortem examination, and was immediately placed in cold storage. As the students had already completed dissection of upper limb, head and neck, thorax, and the greater part of abdomen, it was considered a suitable time for the demonstration, both from their anatomical knowledge and the familiarity with which they regarded embalmed bodies. The subject had been in cold storage for four days when dissection began. The experiment was terminated after $2 \frac{1}{2}$ days, because at the end of this period (despite cold storage when the class was not present) the odour rose above the nuisance level. We are not prepared to comment whether the factor responsible for a tolerable dissecting period, considerably shorter than that customarily employed by our ancestors, was a winter dissecting room temperature of $75^{\circ} \mathrm{F} .\left(24^{\circ}\right.$ C. $)$ or merely a simple lack of intestinal fortitude.

The brunt of demonstration on this cadaver was carried out by the medically qualified members of the gross anatomy teaching group (four out of eight). However, all members of the group (who are always present during scheduled gross anatomy sessions) were available to demonstrate and discuss the unembalmed body during the entire time it was on display. Any student who wished to examine the specimen alone, or in company with his fellows without an instructor, was encouraged to do so. An unlimited supply of disposable surgical gloves was provided for this purpose.

Student comments, derived from a questionnaire were, on the whole, favourable, and $81 \%$ of the 146 students felt that the demonstration was valuable, while $44 \%$ would have liked the experimental scope enlarged to include all regions of the body. Thirty-five per cent. expressed interest, but felt that they had insufficient time to examine the subject fully, owing to upcoming exams and the pressure of work, while only $5 \%$ found the unembalmed body "objectionable" (a similar percentage were "upset" by post mortems in the second semester of the freshmen year) and a final $3 \%$, though not bothered by aesthetic considerations, found it to be "of no value."
These comments suggest that separate regional demonstrations synchronized with student dissections might be an effectiye method for future presentation.-We are, etc.,

N. F. Metcalf

W. K. MEtCalf

D. J. MOFFatT

Department of Anatomy

University of Iowa

U.S.A.

1 Ball, J. M., Andreas Vesalius: The Reformer of Anatomy, p. 75. St. Louis, U.S.A. 1910.

\section{Echovirus Type 4 Infections}

SIR,-Further to the current outbreaks in Tees-side and in Northern Ireland recently referred to (10 July, p. 124), 2 it may be noted from the Weekly Communicable Diseases (Scotland) Reports that this virus is currently also active in Scotland. In this area it was first observed in $1963^{3}$ when it produced an outbreak with an older age distribution than the more common enteroviruses. ${ }^{4}$ Except for a few isolations in 1964 it was not encountered again until 1968 since when only a few strains have been isolated each year until 1971, with 27 isolations reported to date.

In early April, three strains were isolated in Inverness from one family in Ross and Cromarty, but the main focus has been in Lanarkshire, particularly in the Coatbridge/ Airdrie area, with 16 isolations. Other isolations have been reported in recent weeks in Glasgow (five cases) and in Ayrshire (three cases), indicating further spread of infection. In addition, three other cases from Glasgow have been diagnosed by a four-fold rise in antibody titre. In 25 instances, the virus has been isolated from faeces, in one of which isolation was also reported from respiratory secretions. In two cases the virus was obtained from cerebrospinal fluid, in one of which isolation was also reported from a throat swab.

With the exception of three cases, all have suffered from aseptic meningitis, although in every instance as elsewhere recovery has been almost complete within 48 hours and no after effects reported. Of the 30 cases, there have been 16 males and 14 females with a peak incidence in the same 5-20 age groups as in Tees-side and Northern Ireland.

This report was compiled from information provided by contributors to the Weekly C.D.S. Reports.

-I am, etc.

\section{J. C. M. SHARP}

Communicable Diseases (Scotland) Unit.

Ruchill Hospital,

$$
\begin{aligned}
& 1 \text { Lancet, 1971, 2., } 52 . \\
& 2 \text { Donaldson, R. J., Lancet, 1971, 2, } 108 . \\
& 3 \text { Bell, E. I., Lancet, 1964, 1, 1195. } \\
& 4 \text { Landsman, J. B., Bell, E. J., and Grist, N. R., } \\
& \text { Health Bulletin, 1966, 24, 38. }
\end{aligned}
$$

Prophylaxis of Venous Thrombosis

SIR,-Your opening sentence of the leading article "Prophylaxis of Venous Thrombosis" (6 February, p. 305) is, to say the least, timely, and the need of prevention of deep vein thrombosis of the leg and possible fatal thromboembolism extremely urgent. 